\title{
Localized In Vivo Hyperpolarization Transfer Sequences
}

\author{
Mor Mishkovsky, ${ }^{1,2 \star}$ Tian Cheng, ${ }^{1}$ Arnaud Comment, ${ }^{1,3}$ and Rolf Gruetter ${ }^{1,2,4}$
}

\begin{abstract}
In vivo localized and fully adiabatic homonuclear and heteronuclear polarization transfer experiments were designed and performed in the rat brain at 9.4 $\mathrm{T}$ after infusion of hyperpolarized sodium $\left[1,2-{ }^{13} \mathrm{C}_{2}\right]$ and sodium $\left[1-{ }^{13} \mathrm{C}\right]$ acetate. The method presented herein leads to highly enhanced in vivo detection of short- $T_{1}{ }^{13} \mathrm{C}$ as well as attached protons. This indirect detection scheme allows for probing additional molecular sites in hyperpolarized substrates and their metabolites and can thus lead to improved spectral resolution such as in the case of ${ }^{13}$ C-acetate metabolism. Magn Reson Med 68:349-352, 2012. (C) 2011 Wiley Periodicals, Inc.
\end{abstract}

Key words: hyperpolarization; dynamic nuclear polarization; ${ }^{13} \mathrm{C}$ spectroscopy; polarization transfer; brain; metabolism

Hyperpolarization methods designed to enhance the nuclear spin polarization by several orders of magnitude have recently dramatically widened the capability of MR to study biological processes in vivo (1-4). Among the available hyperpolarization schemes, dissolution dynamic nuclear polarization (DNP) is the most versatile method as it can in principle be applied to any molecule (5). Hyperpolarized ${ }^{13} \mathrm{C} \mathrm{MR}$ allows real-time detection of rapid metabolic processes, e.g., several specific enzymecatalyzed reactions have been studied in vitro and in vivo (1). However, the time delay between the DNP process and the in vitro or in vivo MR measurements as well as the finite uptake and metabolic rates of the biologically relevant organisms restrict the application of the technique to nuclear spins with long- $T_{1}$. Consequently, in vitro and in vivo DNP-enhanced metabolic studies have been so far limited to the detection of nonprotonated low- $\gamma$-nuclei such as ${ }^{13} \mathrm{C}$-labeled carbonyls or ${ }^{15} \mathrm{~N}$-labeled quaternary amines. However, to fully determine, understand, or model the biochemical transformations taking place in complex biological systems through isotopomer analysis, it would be useful to probe several molecular sites. For instance, in the case of acetate, the

\footnotetext{
${ }^{1}$ Laboratory for Functional and Metabolic Imaging, Ecole Polytechnique Fédérale de Lausanne, Lausanne, Switzerland.

${ }^{2}$ Department of Radiology, University of Lausanne, Lausanne, Switzerland.

${ }^{3}$ Institute of Condensed Matter Physics, Ecole Polytechnique Fédérale de Lausanne, Lausanne, Switzerland.

${ }^{4}$ Department of Radiology, Geneva University Hospital and Faculty of Medicine, University of Geneva, Geneva 4, Switzerland.

Grant sponsor: Swiss National Science Foundation; Grant numbers: 200020_124901, PP00P2_133562, 31003A_131087; Grant sponsor: National Competence Center in Biomedical Imaging; Grant sponsor: Centre d'Imagerie BioMédicale (CIBM) of the UNIL, UNIGE, HUG, CHUV, EPFL; Grant sponsor: Leenards and Jeantet Foundations.

${ }^{*}$ Correspondence to: Mor Mishkovsky, Ph.D., Laboratory for Functional and Metabolic Imaging (LIFMET), Ecole Polytechnique Fédérale de Lausanne (EPFL), EPFL-SB-IPMC-LIFMET, Station 6, CH-1015 Lausanne (VD), Switzerland. E-mail: mor.mishkovsky@epfl.ch

Received 27 June 2011; revised 22 August 2011; accepted 6 September 2011.

DOI 10.1002/mrm.23231

Published online 21 December 2011 in Wiley Online Library (wileyonlinelibrary.com).

(C) 2011 Wiley Periodicals, Inc.
}

long- $T_{1}$ site of the substrate is the carboxyl position. Unfortunately, the biochemical transformation taking place during $1-{ }^{13} \mathrm{C}$ acetate metabolism via, e.g., tricarboxylic acid (TCA) cycle does not lead to strong transformations of the chemical environment of the ${ }^{13} \mathrm{C}$ label. As a consequence, the chemical shift of the carboxyl ${ }^{13} \mathrm{C}$ is only slightly affected through its transfer from one metabolite to another within the TCA cycle and the following metabolic steps (6). This renders the differentiation between the substrates and its metabolic products difficult. This is particularly challenging in vivo, as the spectral resolution in tissues is more limited than in vitro. Recently, polarization transfer methods were proposed for in vitro DNP-enhanced high-resolution applications in liquid-state NMR allowing the enhancement of short- $T_{1}$ nuclear spins via J-coupling-mediated transfer from the large spin polarization of the long- $T_{1}$ nuclear spins in various molecules (7-9).

In this article, we present an in vivo hyperpolarization transfer sequence to enhance the signal of short- $T_{1}$ nuclear spins. The proposed method allows for the in vivo localized detection of aliphatic carbons or even protons and thus offers the possibility to increase the spectral resolution or obtain additional information on the chemical environment of specific nuclear spins in biomedical hyperpolarized MR experiments. We demonstrate the feasibility and the potential of the method on hyperpolarized acetate in rodent brain.

\section{MATERIALS AND METHODS}

Carbon nuclear spins in a $4.5 \mathrm{M}$ glassy frozen solution of sodium $\left[1,2-{ }^{13} \mathrm{C}_{2}\right]$ acetate or sodium $\left[1-{ }^{13} \mathrm{C}\right]$ acetate $(1: 2$ EtOD: $\mathrm{D}_{2} \mathrm{O}$ v/v containing $30 \mathrm{mM}$ 2,2,6,6-tetramethyl-1piperidinyloxy) were prepared as previously described (10) and were dynamically polarized using a customdesigned DNP polarizer operating at $5 \mathrm{~T}$ and $1 \pm 0.05 \mathrm{~K}$. After $120 \pm 10 \mathrm{~min}$, the polarization reached $13 \pm 1 \%$ and the frozen mixture was rapidly dissolved in $5 \mathrm{~mL}$ of superheated $\left(185 \pm 5^{\circ} \mathrm{C}\right)$ deuterated water and transferred into a plastic infusion pump located inside a 9.4 $\mathrm{T} / 31 \mathrm{~cm}$ actively shielded animal scanner (Magnex; Refs. 11,12). A volume of $2.2 \mathrm{~mL}$ of hyperpolarized solution $(5.2 \mathrm{mmol} / \mathrm{kg}$ ) was infused within $9 \mathrm{~s}$ to Sprague-Dawley rats $(350 \mathrm{~g})$ through a catheter placed in the femoral vein. The animals were anesthetized using $1.5 \%$ isoflurane and their physiology was monitored during the experiments. All experiments were approved by the local ethics committee. Measurements were carried out on an direct drive console (Varian) using a home-built single loop 10-mm-diameter quadrature ${ }^{1} \mathrm{H}$ surface coil with a 10-mm-diameter three loops ${ }^{13} \mathrm{C}$ surface coil (13). In all experiments, the sequence initiation was triggered by an 
Localized homonuclear polarization transfer sequence
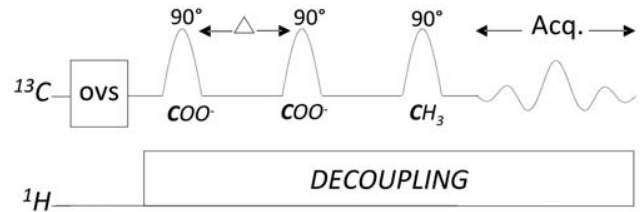

a

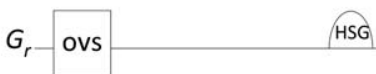

Localized heteronuclear polarization transfer sequence

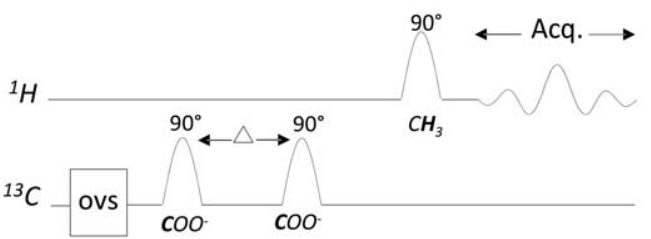

b

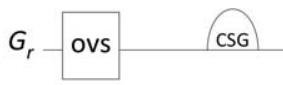

CSG

Evolution of the magnetization

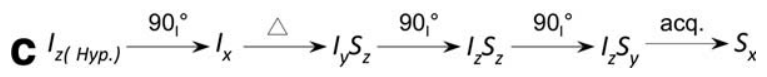

FIG. 1. Sketch of the localized in vivo homonuclear (a) and heteronuclear (b) polarization transfer pulse sequences and evolution of the hyperpolarized magnetization during the sequences presented using the product operator formalism (c). A specific voxel is selected using an outer volume suppression (OVS) block. The enhanced spin polarization located inside the voxel is transferred to the J-coupled nuclear spins prior to detection. In the homonuclear case, a homospoil gradient (HSG) is applied after the second $90^{\circ}$ pulse to destroy residual transverse magnetization. In the heteronuclear case, two coherence selection gradients (CSG) are inserted to suppress the water signal.

external $5 \mathrm{~V}$ signal immediately after infusion, i.e., $12 \mathrm{~s}$ after dissolution. Homonuclear and heteronuclear polarization transfer schemes were designed to acquire DNPenhanced localized spectra of short- $T_{1}$ nuclei in a $9.4 \mathrm{~T}$ using a surface coil for both excitation and detection. Two pulse sequences were implemented (Fig. 1): the first one was designed to transfer the ${ }^{13} \mathrm{C}$ polarization to $\mathrm{J}$ coupled ${ }^{13} \mathrm{C}$ spins and was demonstrated following the infusion of hyperpolarized sodium $\left[1,2-{ }^{13} \mathrm{C}_{2}\right]$ acetate (Fig. 1a); the second one was designed to transfer the ${ }^{13} \mathrm{C}$ polarization to ${ }^{1} \mathrm{H}$ spins via the long distance J-coupling following the infusion of sodium $\left[1-{ }^{13} \mathrm{C}\right]$ acetate (Fig. 1b). Both sequences start with an outer volume suppression block to exclude the signal originating from outside the volume of interest similarly to an approach previously described (14). Subsequently, a modified version of the Insensitive Nuclei Enhanced by Polarization Transfer (INEPT) pulse sequence (15) consisting of three $90^{\circ}$ pulses operating on-resonance is used to transfer the hyperpolarized magnetization localized in a selected single voxel to specific J-coupled short- $T_{1}$ nuclei. The sequence operates as follows: the first $90^{\circ}$ pulse is applied on-resonance on the long- $T_{1}$ nuclear spins, the I spins, and flips the longitudinal hyperpolarized magnet- ization, $\mathrm{I}_{\mathrm{z}}^{\text {hyp }}$, onto the transverse plane yielding a hyperpolarized transverse magnetization $\mathrm{I}_{X}$. This transverse magnetization evolves under its J-coupling ( $\left.J_{\text {IS }}\right)$ with spin $S$ during the delay $\Delta$ to become an (unobservable) antiphase coherence $I_{y} S_{z}$. Following the second $90^{\circ}$ pulse, also applied on the I spins, the magnetization is stored in the $\mathrm{I}_{Z} \mathrm{~S}_{z}$ coherence. The application of the third $90^{\circ}$ pulse, the frequency of which is now set to act on the $S$ spins only, leads to the creation of $\mathrm{I}_{z} \mathrm{~S}_{y}$ coherence. This antiphase coherence evolves under the $J_{\text {IS }}$ coupling during the acquisition time to become detectable in phase $S_{x}$ magnetization which is largely enhanced. To compensate for the $B_{1}$ inhomogeneities inherent to surface coils, hyperbolic-secant adiabatic pulses were used (16). To remove all unwanted residual ${ }^{13} \mathrm{C}$ transverse magnetization in the homonuclear sequence, homospoiled gradients denoted HSG in Fig. 1a were added after the second $90^{\circ}$ pulse. In the homonuclear transfer sequence, due to the strong ${ }^{1} J_{\mathrm{CH}}=127 \mathrm{~Hz}$ that was determined in high resolution (not shown), it is essential to apply proton decoupling to improve the resolution and the signal-to-noise ratio. In addition, the small but non-negligible losses (estimated at $5 \%$ from calculation) due to the ${ }^{2} J_{\mathrm{CH}}=6 \mathrm{~Hz}$ can be avoided by decoupling during the evolution delay $\Delta$ in the homonuclear sequence. To achieve suppression of the strong water signal in the heteronuclear ${ }^{1} \mathrm{H}$ detected sequence, coherence-selective gradients denoted CSG in Fig. 1b were incorporated into the sequence (17).

\section{RESULTS}

Sequence parameters were optimized using a phantom containing sodium $\left[1,2-{ }^{13} \mathrm{C}_{2}\right]$ acetate and sodium $\left[1-{ }^{13} \mathrm{C}\right]$ acetate for the homonuclear and heteronuclear transfers, respectively. The sequences were then applied to acquire the methyl ${ }^{13} \mathrm{C}$ and ${ }^{1} \mathrm{H}$ signal of hyperpolarized acetate

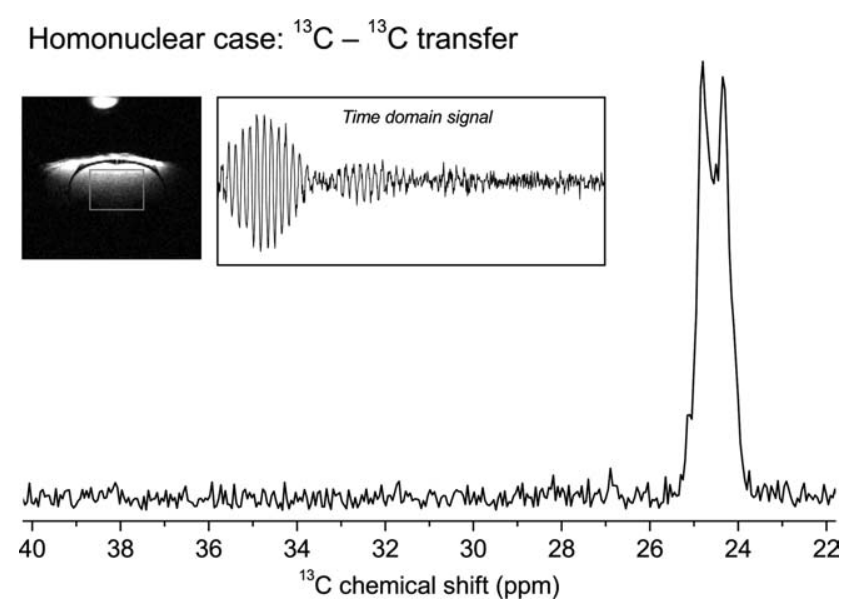

FIG. 2. Magnitude of typical in vivo single scan acquisition following polarization transfer from the carboxyl ${ }^{13} \mathrm{C}$ to the methyl ${ }^{13} \mathrm{C}$ following the infusion of hyperpolarized $[1,2]{ }^{13} \mathrm{C}_{2}$-acetate $(n=4)$. The signal was localized in the area denoted in gray on the ${ }^{1} \mathrm{H}$ image. Hyberbolic secant $500 \mu \mathrm{s}$ adiabatic pulses $\left(B_{1}=5 \mathrm{kHz}\right.$; bandwidth $=3.5 \mathrm{kHz}$ ) were selectively applied on the carboxyl and aliphatic carbons, respectively. The evolution delay $\Delta$ was set to $9.4 \mathrm{~ms}$. $10 \mu \mathrm{s}$ spoil gradient were applied along all three directions $\left(G_{x}=10, G_{y}=16\right.$, and $\left.G_{z}=8.5 \mathrm{G} / \mathrm{cm}\right)$. 
Heteronuclear case: ${ }^{13} \mathrm{C}-{ }^{1} \mathrm{H}$ transfer
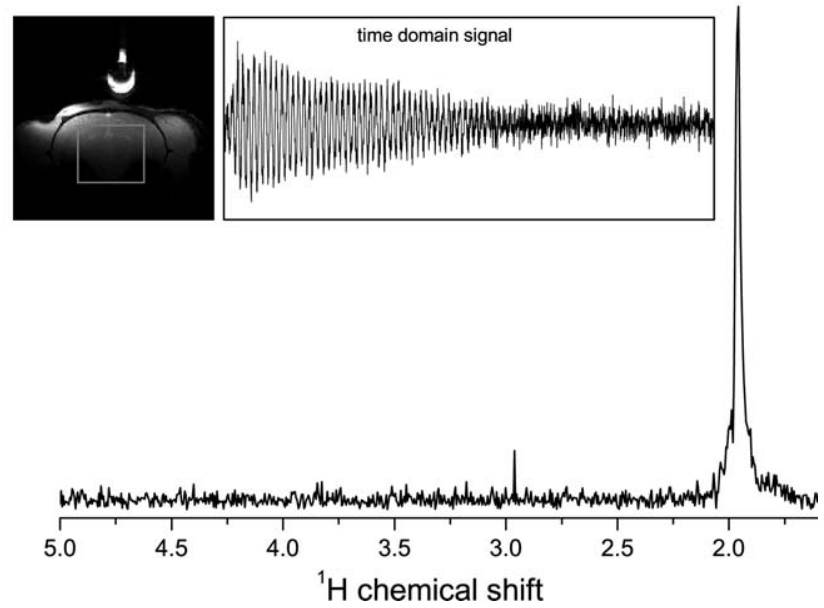

FIG. 3. Magnitude of typical in vivo single scan acquisition following polarization transfer from the carboxyl ${ }^{13} \mathrm{C}$ to the ${ }^{1} \mathrm{H}$ following the infusion of hyperpolarized $1^{13} \mathrm{C}$-acetate $(n=4)$. The signal was localized in the area denoted in gray on the ${ }^{1} \mathrm{H}$ image. Hyberbolic secant $500 \mu \mathrm{s}$ adiabatic pulses $\left(B_{1}=5 \mathrm{kHz}\right.$; bandwidth $=$ $3.5 \mathrm{kHz}$ ) were selectively applied on the carboxyl and methyl protons, respectively. The evolution delay $\Delta$ was set to $40 \mathrm{~ms}$. Note the absence of ${ }^{1} \mathrm{H}$ water signal in the spectrum on account of the coherence-selective gradients which were applied for $10 \mathrm{~ms}$ on the carbon and $2.515 \mathrm{~ms}$ on the proton $\left(G_{x}=20, G_{y}=32\right.$, and $\left.G_{z}=17 \mathrm{G} / \mathrm{cm}\right)$.

in vivo in the rat brain following the infusion of hyperpolarized sodium $\left[1,2-{ }^{13} \mathrm{C}_{2}\right]$ acetate or sodium $\left[1-{ }^{13} \mathrm{C}\right]$ acetate solutions. In the homonuclear $\left({ }^{13} \mathrm{C}-{ }^{13} \mathrm{C}\right)$ case, the intrinsically large chemical shift dispersion of the ${ }^{13} \mathrm{C}$ nuclei allowed to selectively excite the carboxyl carbon without affecting the aliphatic region (and vice versa) due to the limited radiofrequency bandwidth of the adiabatic radiofrequency pulse $(3.5 \mathrm{kHz}) \cdot{ }^{13} \mathrm{C}^{-13} \mathrm{C}$ polarization transfer was achieved via the ${ }^{1} J_{\mathrm{CC}}$ coupling $\left({ }^{1} J_{\mathrm{CC}}=\right.$ $52 \mathrm{~Hz}$ ) in sodium $\left[1,2-{ }^{13} \mathrm{C}_{2}\right]$ acetate by setting the delay $\Delta$ between the two first $90^{\circ}$ pulses equal to $1 / 2 J_{\mathrm{cc}}$ (see Fig. 1a). The time domain signal (free induction decay) following the last pulse starts from the nondetectable $\mathrm{I}_{z} \mathrm{~S}_{y}$ two-spin antiphase coherence and evolves during the acquisition to become a detectable in phase term, namely $S_{x}$. The sine-like envelope of the free induction decay results in an antiphase frequency spectrum (Fig. 2).

In the heteronuclear ${ }^{13} \mathrm{C}-{ }^{1} \mathrm{H}$ experiments, the polarization transfer was performed through the weak but nonnegligible ${ }^{2} J_{\mathrm{CH}}$ coupling $\left({ }^{2} J_{\mathrm{CH}}=6 \mathrm{~Hz}\right)$ between the carboxyl carbon and the methyl protons in sodium $\left[1-{ }^{13} \mathrm{C}\right]$ acetate. Spin multiplicity of the three equivalent methyl protons coupled to the carboxyl carbon has to be taken into account when setting the $\Delta$ delay between the two first $90^{\circ}$ pulses (see Fig. 1b). As in the homonuclear case, the free induction decay starts from a nondetectable two-spin antiphase coherence which develops during the acquisition to become a detectable coherence (Fig. 1c). A typical ${ }^{1} \mathrm{H}$ magnitude spectrum is presented in Fig. 3. Note the absence of ${ }^{1} \mathrm{H}$ water signal in the spectrum which was eliminated by the coherence-selective gradients (Fig. 1).

\section{DISCUSSION}

The sequences presented herein, which are compatible with the use of surface coils, are designed for in vivo metabolic studies with molecules containing short- $T_{1}$ nuclear spin sites coupled to long-lived hyperpolarized nuclear spins via J-coupling. Thus, additional spectroscopic information from different chemical sites can be recorded, e.g., to assert the assignment of metabolites detected in hyperpolarized MR experiments. Furthermore, this type of detection can lead to improved spectral resolution by probing different nuclear spins of hyperpolarized precursors and their metabolites.

The transfer scheme presented above leads to unwanted but unavoidable signal losses due to the finite in vivo transverse relaxation. During the $\Delta$ delay following the first pulse, the loss of spin coherence is governed by relaxation processes described by the carboxyl ${ }^{13} \mathrm{C} T_{2}^{*}$ decay constant. A value of $T_{2}^{*}=53 \mathrm{~ms}$ was deduced from the full width at half maximum $=6 \mathrm{~Hz}$ of hyperpolarized sodium $\left[1-{ }^{13} \mathrm{C}\right]$ acetate localized ${ }^{13} \mathrm{C}$ spectra measured in vivo (data not shown) using the relation $T_{2}^{*}=\frac{1}{\pi \cdot \text { FWHM }}$. Taking into account that the transverse magnetization decays according to $e^{-\Delta / T_{2}^{*}}$, an estimated $16 \%$ of the ${ }^{13} \mathrm{C}$ polarization is lost after $\Delta=9.4 \mathrm{~ms}$ in the ${ }^{13} \mathrm{C}^{13} \mathrm{C}$ homonuclear case. The losses are about $50 \%$ in the heteronuclear ${ }^{13} \mathrm{C}^{-1} \mathrm{H}$ case as the delay corresponding to the weak $J_{\mathrm{CH}}=6 \mathrm{~Hz}$ is set to $\Delta=40 \mathrm{~ms}$. Additional losses occur during acquisition time as the evolution of the antiphase $I_{z} S_{y}$ coherence that is converted into the observable $S_{x}$ coherence is also subject to $T_{2}^{*}$ relaxation. With a $T_{2}^{*}$ of $22 \mathrm{~ms}$ deduced from the linewidth of ${ }^{1} \mathrm{H}$ spectrum (Fig. 3), the overall signal losses in the heteronuclear experiment were estimated to be about $90 \%$, meaning that only $10 \%$ of the enhanced ${ }^{13} \mathrm{C}$ polarization survives the polarization transfer sequence. The consequence of signal losses of magnetic field inhomogeneities could be recovered by incorporating two simultaneous $180^{\circ}$ pulses on both ${ }^{13} \mathrm{C}$ and ${ }^{1} \mathrm{H}$ channels at a time $\Delta / 2$ after the initial ${ }^{13} \mathrm{C} 90^{\circ}$ pulse as well as after the ${ }^{1} \mathrm{H}$ $90^{\circ}$ pulse. The loss of spin coherence would then be governed by $T_{2}$ instead of $T_{2}^{*}$, but the additional pulses might also lead to a degradation of the signal intensity especially if adiabatic pulses of non-negligible length are used. Finally, it is worth mentioning that coherence selective gradients refocus only half of the signal and in addition diffusion effects linked to the application of the coherence selection gradients can also lead to minor signal losses in the heteronuclear ${ }^{13} \mathrm{C}-{ }^{1} \mathrm{H}$ detection scheme.

Hyperpolarized MR experiments with two particular substrates, namely ${ }^{13} \mathrm{C}$-acetate (18) and ${ }^{15} \mathrm{~N}$-choline (19) both of which having already been used for in vivo hyperpolarized studies, could benefit from the technique presented herein. For these two DNP precursors, the biochemical transformations of interest do not involve the chemical bonds directly attached to the long- $T_{1}$ nuclear spins. Consequently, the observed chemical shift variations between the precursor long- $T_{1}$ sites and the corresponding sites in the metabolites are likely to be small. The standard direct detection scheme may thus be limited in terms of spectral resolution and the precursors and metabolites spectra might overlap, especially at 
low field. In specific cases, e.g., methyl ${ }^{13} \mathrm{C}$ or ${ }^{1} \mathrm{H}$ in ${ }^{13} \mathrm{C}$-acetate or ${ }^{1} \mathrm{H}$ in ${ }^{15} \mathrm{~N}$-choline and their respective metabolites $(7,9)$, larger chemical shift changes can be detected at other chemical sites.

\section{CONCLUSION}

The pulse sequences presented in this note consist of only three radiofrequency pulses and are thus simple to implement for in vivo applications. The proposed sequences are also compatible with surface coils as adiabatic pulses can be applied. The indirect detection schemes presented herein provides an alternative means to detect in vivo transformations using DNP-enhanced MR and extends the in vivo applicability of hyperpolarized MR to short- $T_{1}$ nuclear spins, in particular protons.

\section{REFERENCES}

1. Gallagher FA, Kettunen MI, Brindle KM. Biomedical applications of hyperpolarized C-13 magnetic resonance imaging. Prog Nucl Magn Reson Spectrosc 2009;55:285-295.

2. Kurhanewicz J, Vigneron DB, Brindle KM, Chekmenev EY, Comment A, Cunningham CH, DeBerardinis RJ, Green GG, Leach MO, Rajan SS, Rizi RR, Ross BD, Warren WS, Malloy CR. Analysis of cancer metabolism by imaging hyperpolarized nuclei: prospects for translation to clinical research. Neoplasia 2011;13:81-97.

3. Oros AM, Shah NJ. Hyperpolarized xenon in NMR and MRI. Phys Med Biol 2004;49:R105-R153.

4. Viale A, Reineri F, Santelia D, Cerutti E, Ellena S, Gobetto R, Aime S. Hyperpolarized agents for advanced MRI investigations. Q J Nucl Med Mol Imaging 2009;53:604-617.

5. Ardenkjaer-Larsen JH, Fridlund B, Gram A, Hansson G, Hansson L, Lerche MH, Servin R, Thaning M, Golman K. Increase in signal-tonoise ratio of $>10,000$ times in liquid-state NMR. Proc Natl Acad Sci USA 2003;100:10158-10163.

6. Fan WMT. Metabolite profiling by one- and two-dimensional NMR analysis of complex mixtures. Prog Nucl Magn Reson Spectrosc 1996;28:161-219.
7. Harris T, Giraudeau P, Frydman L. Kinetics from indirectly detected hyperpolarized NMR spectroscopy by using spatially selective coherence transfers. Chem-A Eur J 2011;17:697-703.

8. Mishkovsky M, Frydman L. Progress in hyperpolarized ultrafast 2D NMR spectroscopy. ChemPhysChem 2008;9:2340-2348.

9. Sarkar R, Comment A, Vasos PR, Jannin S, Gruetter R, Bodenhausen G, Hall H, Kirik D, Denisov VP. Proton NMR of N-15-choline metabolites enhanced by dynamic nuclear polarization. J Am Chem Soc 2009;131:16014-16015.

10. Kurdzesau F, van den Brandt B, Comment A, Hautle P, Jannin S, van der Klink JJ, Konter JA. Dynamic nuclear polarization of small labelled molecules in frozen water-alcohol solutions. J Phys D-Appl Phys 2008;41:155506.

11. Comment A, van den Brandt B, Uffmann K, Kurdzesau F, Jannin S, Konter JA, Hautle P, Wenckebach WT, Gruetter R, van der Klink JJ. Design and performance of a DNP prepolarizer coupled to a rodent MRI scanner. Concepts Magn Reson Part B: Magn Reson Eng 2007; 31B:255-269.

12. Jannin S, Comment A, Kurdzesau F, Konter JA, Hautle P, van den Brandt B, van der Klink JJ. A $140 \mathrm{GHz}$ prepolarizer for dissolution dynamic nuclear polarization. J Chem Phys 2008;128:241102.

13. Adriany G, Gruetter R. A half-volume coil for efficient proton decoupling in humans at 4 Tesla. J Magn Reson 1997;125:178-184.

14. Choi IY, Tkac I, Gruetter R. Single-shot, three-dimensional "nonecho" localization method for in vivo NMR spectroscopy. Magn Reson Med 2000;44:387-394.

15. Morris GA, Freeman R. Enhancement of nuclear magnetic-resonance signals by polarization transfer. J Am Chem Soc 1979;101: $760-762$.

16. Silver MS, Joseph RI, Hoult DI. Highly selective $\mathrm{Pi} / 2$ and Pi-pulse generation. J Magn Reson 1984;59:347-351.

17. Bodenhausen G, Kogler H, Ernst RR. Selection of coherence-transfer pathways in NMR pulse experiments. J Magn Reson 1984;58: 370-388.

18. Mishkovsky M, Comment A, Gruetter R. In vivo detection of brain Krebs cycle intermediate by hyperpolarized MR. In: Proc Int Soc Mag Reson Med, Montreal, Canada, vol. 19, 2011. p 660.

19. Cudalbu C, Comment A, Kurdzesau F, van Heeswijk RB, Uffmann K, Jannin S, Denisov V, Kirik D, Gruetter R. Feasibility of in vivo N-15 MRS detection of hyperpolarized N-15 labeled choline in rats. Phys Chem Chem Phys 2010;12:5818-5823. 